American J. of Engineering and Applied Sciences 4 (4): 491-496, 2011

ISSN 1941-7020

(C) 2014 L. Benmesbah et al., This open access article is distributed under a Creative Commons Attribution

(CC-BY) 3.0 license

\title{
Optimal Decentralized Spectral Resource Allocation for Orthogonal Frequency Division Multiple Access Downlink of Femto Networks Via Adaptive Gradient Vector Step Size Approach
}

\author{
${ }^{1}$ Lamia Benmesbah, ${ }^{2}$ Bingo Wing-Kuen Ling, \\ ${ }^{3}$ Vikram Chandrasekhar, ${ }^{1}$ Xiaoli Chu and ${ }^{4}$ Mischa Dohler \\ ${ }^{1}$ Department of Electronic Engineering, Division of Engineering, \\ King's College London, Strand, London, WC2R 2LS, United Kingdom \\ ${ }^{2}$ School of Engineering, University of Lincoln, \\ Lincoln, Lincolnshire, LN6 7TS, United Kingdom \\ ${ }^{3}$ Department of Electrical and Computer Engineering, University of Texas at Austin, \\ ${ }^{1}$ University Station, Austin, Texas 78712, United States of America \\ ${ }^{4}$ Centre Tecnologic de Telecomunicacions de Catalunya, \\ Parc Mediterrani de la Tecnologia, Building B4, Av. Carl Friedrich Gauss 7, \\ 08860 Castelldefels, Barcelona, Spain
}

\begin{abstract}
Problem statement: For the Orthogonal Frequency Division Multiple Access (OFDMA) downlink of a femto network, the resource allocation scheme would aim to maximize the Area Spectral Efficiency (ASE) subject to constraints on the radio resources per transmission interval accessible by each femtocell. Approach: An optimal resource allocation scheme for completely decentralized femtocell deployments leads to a nonlinear optimization problem because the cost function of the optimization problem is nonlinear. In this study, an adaptive gradient vector step size approach is proposed for finding the optimal solution of the optimization problem. Results: Computer numerical simulation results show that our proposed method is more efficient than existing exhaustive search methods. Conclusion: By adpative changing the step size of the gradient vector, the locally optimal solution can be achieved quickly.
\end{abstract}

Key words: Quadrature Amplitude Modulations (QAMs), mobile User Equipments (UEs), Orthogonal Frequency Division Multiple Access (OFDMA), Area Spectral Efficiency (ASE), Femtocell Access Points (FAPs), Signal to Interference Ratio (SIR), spectral resource

\section{INTRODUCTION}

Mobile User Equipments (UEs) have become increasingly important in our daily lives and mobile operators have been spending a lot of money and efforts to meet the growing customer demands. However, most current cellular networks suffer from poor indoor network coverage and cellsite usage saturation. As a consequence, mobile operators face poor customer retain-ability and increasing churn. One cost-effective solution for mobile operators to improve coverage and offload macrocell traffic as well as to reduce churn is the emerging femtocell paradigm. Typically, a large number of Femtocell Access Points (FAPs) (Yeh et al., 2008) are overlaid on macrocells. Each FAP provides high data-rate connections to UEs within a short range using the same radio access technology as the microcell underlay.

A pertinent challenge for mobile operators is the management of interference between neighboring femtocells. As FAPs are likely to be deployed by end users, the total number and locations of active FAPs is a priori unknown to mobile operators. Therefore, interference caused by femtocells cannot be managed using conventional network planning methods and interference avoidance strategies are preferred over mutual interference suppression strategies for femtocells (Chandrasekhar et al., 2008). On the other hand, intercell interference in the hierarchical macro/femto network is closely related to the femtocell access policy, which defines the way how a femtocell allows or restricts its usage to users (Chandrasekhar et $a l .$, 2008). This study will focus on the closed access 
femtocell, which serves a group of authorized UEs only, since the closed access policy would be preferred by subscribers of the femtocell.

As OFDMA has been considered in the downlink for next generation wireless networks 3GPP LTE IEEE 802 2007, OFDMA based femtocells are widely expected to deliver massive improvements in coverage and capacity (Yeh et al., 2008). To mitigate intercell interference, frequency reuse (Stolyar and Viswanathan, 2008; Elayoubi et al., 2008; Assaad and Mourad, 2008) and intercell coordination (Necker, 2007; Simonsson, 2007) schemes have been studied in OFDMA macro networks. A centralized downlink frequency planning across femtocells and macrocells was proposed in Lopez-Perez et al. (2008), but the large number of femtocells significantly complicates the centralized optimization process.

The spectrum allocation policy in (Chandrasekhar and Andrews, 2009) avoids cross-tier interference by assigning orthogonal spectrum resources to the macrocell and femtocell tiers and diminishes femto-tofemto interference by allowing each femtocell to access only a random subset of the spectrum resources that are assigned to the femtocell tier. As a result, the average number of interfering femtocells in each frequency subchannel is reduced. The portion of spectrum resources accessible by each femtocell is determined based on optimizing the throughput per cell and ASE (Chandrasekhar and Andrews, 2009). To optimize such a decentralized resource allocation leads to an optimization problem for which the cost function is nonlinear. Although the nonlinear optimization problem was solved by exhaustive search methods (Chandrasekhar and Andrews, 2009; Chandrasekhar et al., 2009), the computational costs of which are very high. In this study we propose an adaptive gradient vector step size approach for finding the optimal solution of the nonlinear optimization problem so that the computational costs could be reduced.

Problem formulation: We consider the OFDMA downlink of a macro/femto cellular network. Closed access femtocells are randomly overlaid on the microcell and the locations of FAPs form a Stationary Poisson Point Process (SPPP) on $\mathfrak{R}^{2}$ (Weber et al., 2007). It is assumed that microcells and femtocells in the network are allocated with orthogonal spectrum resources and there is no macro-to-femto or femto-tomacro interference. This study focuses on the decentralized resource allocation between femtocells only. Denote the radius of a hexagonal microcell and the spatial intensity of FAPs as $r_{M}$ and $\lambda_{F}$, respectively, where $\lambda_{F}$ is defined as the average number of femtocells per unit area. Denote the radius of each femtocell and the number of indoor UEs to be served by each femtocell as $r_{F}$ and $U_{F}$, respectively, where it is assumed that they are the same for all femtocells. Denote the average number of FAPs per microcell as $\mathrm{N}_{\mathrm{F}}$. Since cellular networks are typically interference limited, thermal noise at the receiver is neglected in this study.

The basic resource unit that is addressable for OFDMA transmissions is a Resource Block (RB), which is a two dimensional block defined in the time and frequency domains. In the third Generation Partnership Project (3GPP) Long Term Evolution (LTE) release 8 3GPP Release 8 , an RB has a time duration of $1 \mathrm{~ms}$ and spans either 12 subcarriers with a subcarrier bandwidth of $15 \mathrm{kHz}$ or 24 subcarriers with a subcarrier bandwidth of $7.5 \mathrm{kHz}$. Intracellular interference is avoided by maintaining the orthogonality among co-cell UEs in OFDMA networks (Andrews, 2008), i.e., maintaining one scheduled UE per RB in each cell. Not to clutter subsequent analysis, it is assumed that an FAP assigns equal transmission power all over RBs for a given transmission interval.

Let the total number of distinct RBs available to all femtocells for each transmission time interval be $F$ $\left(\in \mathrm{Z}^{+}\right)$. Each FAP is allowed to use only $\mathrm{K}\left(\in \mathrm{Z}^{+}, \mathrm{K} \leq \mathrm{F}\right)$ RBs for downlink transmissions in each transmission time interval. For ease of implementation, the value of $\mathrm{K}$ is assumed to be the same for all femtocells. Define the fraction of radio resources per transmission interval accessible by each femtocell as $\rho_{\mathrm{F}}$ and we have Eq. 1:

$$
\rho_{\mathrm{F}}=\frac{\mathrm{K}}{\mathrm{F}}
$$

where, $0 \leq \rho_{\mathrm{F}} \leq 1$. When $\rho_{\mathrm{F}}=1$, each femtocell can access all the available RBs.

If each femtocell chooses the $\mathrm{K}$ distinct $\mathrm{RBs}$ independently and with equal probability, then the probability of a femtocell selecting a given RB for transmission is $\rho_{\mathrm{F}}$. This implies that the average number of interfering femtocells in each $R B$ is effectively reduced and the femto-to-femto interference is diminished.

For a given RB, denote the received Signal to Interference Ratio (SIR) of a femto UE as SIR $_{F}$, the distance from an interfering FAP $_{\mathrm{i}}$ to the femto UE of interest as $\left|\mathrm{X}_{\mathrm{Fi}}\right|$, the path loss exponent on the link from the home FAP to the femto UE as $\alpha_{\mathrm{F}}$, the path loss exponent on the link from an interfering $\mathrm{FAP}_{\mathrm{i}}$ to the femto UE as $\alpha_{\mathrm{FF}}$, the channel power gain between the home FAP and the femto UE as $\mathrm{H}_{0} \sim \operatorname{EXP}(1)$ (unit mean 
exponential distribution), the channel power gain between an interfering $\mathrm{FAP}_{\mathrm{i}}$ and the femto UE as $\mathrm{H}_{0 \mathrm{i}} \sim \mathrm{EXP}(1)$, the indoor lognormal shadowing on the link from the home FAP to the femto UE as $\Theta_{0}$ and the lognormal shadowing on the link from an interfering FAP $_{\mathrm{i}}$ to the femto UE as $\Theta_{0 \mathrm{i}}$. It is assumed that all $\mathrm{H}_{0}$, $\mathrm{H}_{0 \mathrm{i}}, \Theta_{0}$ and $\Theta_{0 \mathrm{i}}$ are mutually independent. Let the mean and the standard deviation of $\Theta_{0}$ be $\mu$ and $\sigma$, respectively and those of $\Theta_{0 \mathrm{i}}$ be $\mu_{\mathrm{i}}$ and $\sigma_{\mathrm{i}}$, respectively, i.e., $\Theta_{0} \sim \mathrm{LN}\left(\zeta \mu, \zeta^{2} \sigma^{2}\right)$ and $\Theta_{0 \mathrm{i}} \sim \mathrm{LN}\left(\zeta \mu_{\mathrm{i}}, \zeta^{2} \sigma_{\mathrm{i}}^{2}\right)$, where all $\Theta_{0 \mathrm{i}}$ are i.i.d. and $\zeta \equiv 0.1 \ln 10$ is a scaling constant (Chandrasekhar and Andrews, 2009). Denote the single-wall penetration loss as $\phi$. Consider the case of double-wall penetration losses for links from an interfering FAP to the femto UE. The worst case scenario that all femto UEs are located on the edge of their home femtocells is assumed. Denote the index set corresponding to all $\mathrm{FAP}_{\mathrm{i}}$ that have access to the given $\mathrm{RB}$ as $\Phi$, then we have Eq. 2:

$$
\operatorname{SIR}_{\mathrm{F}}=\frac{\Theta_{0} \mathrm{H}_{0} \mathrm{r}_{\mathrm{F}}^{-\alpha_{\mathrm{F}}}}{\sum_{\mathrm{i} \in \Phi} \Theta_{0 \mathrm{i}} \varphi^{2} \mathrm{H}_{0 \mathrm{i}}\left|\mathrm{X}_{\mathrm{Fi}}\right|^{-\alpha_{\mathrm{FF}}}}
$$

The product of a lognormal shadowing and an exponential fading coefficient can be modeled as a lognormal distribution using Turkmani (1992) approximation. That is, $\Theta_{0} \mathrm{H}_{0}$ is approximated by a lognormal random variable denoted as $\psi_{0} \sim \mathrm{LN}\left(\mu^{\prime}, \sigma^{\prime 2}\right)$, where $\mu^{\prime}=\zeta(\mu-2.5)$ and $\sigma^{\prime}=\zeta \sqrt{\sigma^{2}+5.57^{2}}$. Similarly, each $\Theta_{\mathrm{i}} \mathrm{H}_{\mathrm{i}}$ is approximated by a lognormal random variable denoted as $\psi_{0 \mathrm{i}^{2}} \mathrm{LN}\left(\mu_{\mathrm{i}}^{\prime}, \quad \sigma_{\mathrm{i}}{ }^{\prime 2}\right)$, where $\mu_{\mathrm{i}}^{\prime}=\zeta\left(\mu_{\mathrm{i}}-2.5\right)$ and $\sigma_{i}^{\prime}=\zeta \sqrt{\sigma_{i}^{2}+5.57^{2}}$. Then, (2) can be simplified to Eq. 3:

$$
\operatorname{SIR}_{\mathrm{F}}=\frac{\psi_{0} \mathrm{r}_{\mathrm{F}}^{-\alpha_{\mathrm{F}}}}{\sum_{\mathrm{i} \in \Phi} \psi_{0 i} \varphi^{2}\left|\mathrm{X}_{\mathrm{Fi}}\right|^{-\alpha_{\mathrm{FF}}}}
$$

Define the boundary of the SIR as $\Gamma$. Based on (3), the Cumulative Distribution Function (CDF) of the SIR of a femto UE can be calculated as Eq. 4:

$$
\begin{aligned}
& \operatorname{Pr}\left(\operatorname{SIR}_{\mathrm{F}} \leq \Gamma\right)=\operatorname{Pr}\left(\frac{\psi_{0} \mathrm{r}_{\mathrm{F}}^{-\alpha_{\mathrm{F}}}}{\sum_{\mathrm{i} \in \Phi} \psi_{0 \mathrm{i}} \varphi^{2}\left|X_{\mathrm{Fi}}\right|^{-\alpha_{\mathrm{FF}}}} \leq \Gamma\right) \\
& =E_{\psi_{0}}\left(\operatorname{Pr}\left(\sum_{i \in \Phi} \psi_{0 i} \varphi^{2}\left|X_{F_{i}}\right|^{-\alpha_{F F}} \geq\left.\frac{\psi_{0} \mathrm{r}_{\mathrm{F}}^{-\alpha_{F}}}{\Gamma}\right|_{\psi_{0}}\right)\right) \\
& \geq 1-\mathrm{E}_{\psi_{0}}\left(\exp \left(-\pi \lambda_{\mathrm{F}} \rho_{\mathrm{F}} \mathrm{E}\left(\psi_{\mathrm{I}}^{\delta_{\mathrm{t}}}\right)\left(\frac{\Gamma}{\psi_{0} \mathrm{r}_{\mathrm{F}}^{-\alpha_{\mathrm{F}}}}\right)^{\delta_{\mathrm{f}}}\right)\right) \\
& =1-\mathrm{E}_{\psi_{0}}\left(\exp \left(-\rho_{\mathrm{F}} \mathrm{k}_{\mathrm{f}} \Gamma^{\delta_{\mathrm{f}}} \Psi_{0}^{-\delta_{\mathrm{f}}}\right)\right)
\end{aligned}
$$

where, $\delta_{\mathrm{f}} \equiv \frac{2}{\alpha_{\mathrm{FF}}}, \psi_{\mathrm{I}}$ is the equivalent value of $\Psi_{\mathrm{i}} \forall_{\mathrm{i} \in \Phi}$, $\mathrm{k}_{\mathrm{f}} \equiv \pi \lambda_{\mathrm{F}} \mathrm{E}\left(\psi_{\mathrm{I}}^{\delta_{\mathrm{r}}}\right) \mathrm{r}_{\mathrm{F}}^{\alpha_{\mathrm{F}} \delta_{\mathrm{r}}}, \quad \mathrm{E}_{\psi_{\mathrm{f}}}(\cdot)$ and $\mathrm{E}\left(\psi_{\mathrm{I}}^{\delta_{\mathrm{f}}}\right)$ are the expected values with respect to $\psi_{0}$ and $\psi_{\mathrm{I}}$, respectively.
Define the total number of discrete rates that an adaptive modulation realizes as $\mathrm{L}\left(\in \mathrm{Z}^{+}\right)$, the Shannon gap of Quadrature Amplitude Modulations (QAMs) as $\Psi$, the boundaries of the SIR ranges as $\Gamma_{l}$ for $l \in\{1, \ldots, \mathrm{L}\}$ and the transmission rates as $b_{1}$ for $l \in\{1, \ldots, L\}$. When the UE received SIR lies in $\left[\Gamma_{1}, \Gamma_{1+1}\right)$ for $l \in\{1, \ldots, \mathrm{L}\}$, the FAP decides the transmission rate according to Eq. 5:

$\mathrm{b}_{1}=\log _{2}\left(1+\frac{\Gamma_{1}}{\Psi}\right),(\mathrm{b} / \mathrm{s} / \mathrm{Hz})$

With $b_{1}=1$ for $1=1,2 \ldots, L$, the expected throughput of a femtocell is given by Eq. 6 :

$$
\begin{aligned}
& \mathrm{T}_{\mathrm{f}}=\sum_{\mathrm{l}=1}^{\mathrm{L}-1} \operatorname{lPr}\left(\Gamma_{\mathrm{l}} \leq \mathrm{SIR}_{\mathrm{F}}<\Gamma_{\mathrm{l}+1}\right)+\mathrm{L}_{\mathrm{Pr}}\left(\operatorname{SIR}_{\mathrm{F}} \geq \Gamma_{\mathrm{l}}\right) \\
& =\sum_{\mathrm{l}=1}^{\mathrm{L}-1} \mathrm{IE}_{\Psi_{0}}\left[\exp \left(-\rho_{\mathrm{F}} \mathrm{k}_{\mathrm{f}} \Gamma_{\mathrm{l}}^{\delta_{\mathrm{f}}} \Psi_{0}^{-\delta_{\mathrm{f}}}\right)-\exp \left(\begin{array}{l}
-\rho_{\mathrm{F}} \mathrm{k}_{\mathrm{f}} \\
\Gamma_{\mathrm{l}+1}^{\delta_{\mathrm{f}}} \Psi_{0}^{-\delta_{\mathrm{f}}}
\end{array}\right)\right] \\
& +\mathrm{LE} \Psi_{0}\left[\exp \left(-\rho_{\mathrm{F}} \mathrm{k}_{\mathrm{f}} \Gamma_{1}^{\delta_{\mathrm{f}}} \Psi_{0}^{-\delta_{\mathrm{f}}}\right)\right] \\
& =\mathrm{E} \Psi_{0}\left[\sum_{\mathrm{l}=1}^{\mathrm{L}} \operatorname{lexp}\left(-\rho_{\mathrm{F}} \mathrm{k}_{\mathrm{f}} \Gamma_{\mathrm{l}}^{\delta_{\mathrm{f}}} \Psi_{0}^{-\delta_{\mathrm{f}}}\right)-\sum_{\mathrm{l}=1}^{\mathrm{L}-1} \operatorname{lexp}\left(\begin{array}{l}
-\rho_{\mathrm{F}} \mathrm{k}_{\mathrm{f}} \\
\Gamma_{\mathrm{l}+1}^{\delta_{\mathrm{f}}} \Psi_{0}^{-\delta_{\mathrm{f}}}
\end{array}\right)\right] \\
& =\mathrm{E} \Psi_{0}\left[\sum_{\mathrm{l}=1}^{\mathrm{L}} \operatorname{lexp}\left(-\rho_{\mathrm{F}} \mathrm{k}_{\mathrm{f}} \Gamma_{\mathrm{l}}^{\delta_{\mathrm{f}}} \Psi_{0}^{-\delta_{\mathrm{f}}}\right)\right]
\end{aligned}
$$

Then the ASE offered by the femtocell network is given by Eq. 7:

$$
\begin{aligned}
& \operatorname{ASE}_{\mathrm{F}}\left(\rho_{\mathrm{F}}\right)=\rho_{\mathrm{F}} \lambda_{\mathrm{F}} \mathrm{T}_{\mathrm{f}} \\
& =\rho_{\mathrm{F}} \lambda_{\mathrm{F}} \mathrm{E}_{\psi_{0}}\left[\sum_{l=1}^{\mathrm{L}} \exp \left(-\rho_{\mathrm{F}} \mathrm{k}_{\mathrm{f}} \Gamma_{1}^{\delta_{t}} \psi_{0}^{-\delta_{t}}\right)\right] \\
& =\rho_{\mathrm{F}} \lambda_{\mathrm{F}} \int_{0}^{+\infty}\left[\sum_{l=1}^{\mathrm{L}} \exp \left(-\rho_{\mathrm{F}} \mathrm{k}_{\mathrm{f}} \Gamma_{1}^{\delta_{\mathrm{r}}} \psi_{0}^{-\delta_{\mathrm{t}}}\right)\right] \mathrm{p}\left(\psi_{0}\right) \mathrm{d} \psi_{0}
\end{aligned}
$$

where, $p\left(\psi_{0}\right)=\frac{1}{\psi_{0} \sigma^{\prime} \sqrt{2 \pi}} \exp \left(-\frac{\left(\ln \left(\psi_{0}\right)-\mu^{\prime}\right)^{2}}{2 \sigma^{\prime 2}}\right)$ is the PDF of $\psi_{0}$. Hence, the decentralized spectrum resource allocation that maximizes the $\mathrm{ASE}_{\mathrm{F}}$ can be formulated as the following optimization problem, Eq. 8a and 8b:

\section{$\operatorname{Problem}\left(\mathbf{P}^{\prime}\right)$ :}

$$
\begin{aligned}
& \max _{\rho_{\mathrm{F}}} \operatorname{ASE}_{\mathrm{F}}\left(\rho_{\mathrm{F}}\right) \\
& \text { subject to } \quad 0<\rho_{\mathrm{F}} \leq 1
\end{aligned}
$$

This maximization problem is equivalent to the following Eq. 9a and 9b minimization problem:

\section{Problem ( P ):}

$$
\max _{\rho_{\mathrm{F}}}-\operatorname{ASE}_{\mathrm{F}}\left(\rho_{\mathrm{F}}\right)
$$


Am. J. Engg. \& Applied Sci., 4 (4): 491-496, 2011

subject to $0<\rho_{\mathrm{F}} \leq 1$

\section{MATERIALS AND METHODS}

Adaptive gradient vector step size approach: According to (7), Problem (P) is a nonlinear optimization problem. Exhaustive search methods are used for finding the optimal solution of Problem (P) (Chandrasekhar and Andrews, 2009; Chandrasekhar et al., 2009). However, the computational costs of exhaustive search methods are very high (Chandrasekhar and Andrews, 2009; Chandrasekhar et al., 2009). In order to solve the optimization problem efficiently, we propose the following method for finding the optimal solution of Problem (P).

\section{Algorithm:}

Step 1: Initialize $\mathrm{k}=1, \rho_{1} \in[0,1], \varepsilon>0, \mathrm{D}>1$ and $\alpha_{1}>\varepsilon$.

Step 2: Case I: If $\alpha_{k} \geq \varepsilon$ and $-\operatorname{ASE}_{\mathrm{F}}\left(\rho_{\mathrm{k}}+\left.\alpha_{\mathrm{k}} \frac{\partial \operatorname{ASE}_{\mathrm{F}}(\rho)}{\partial \rho}\right|_{\rho=\rho_{\mathrm{k}}}\right) \leq-\operatorname{ASE}_{\mathrm{F}}\left(\rho_{\mathrm{k}}\right)$, where

$\frac{\partial}{\partial \rho_{\mathrm{F}}} \operatorname{ASE}_{\mathrm{F}}\left(\rho_{\mathrm{F}}\right)$

$=\int_{0}^{+\infty} \frac{\lambda_{\mathrm{F}}}{\psi_{0} \sigma^{\prime} \sqrt{2 \pi}} \exp \left(-\frac{\left(\ln \left(\psi_{0}\right)-\mu^{\prime}\right)^{2}}{2 \sigma^{\prime 2}}\right) \sum_{i=1}^{L}\left\{\frac{\partial}{\partial \rho_{\mathrm{F}}}\left[\rho_{\mathrm{F}} \exp \left(-\rho_{\mathrm{F}} \mathrm{k}_{\mathrm{f}} \Gamma_{1}^{\delta_{t}} \psi_{0}^{-\delta_{t}}\right)\right]\right\} \mathrm{d} \psi_{0}$

$=\int_{0}^{+\infty} \frac{\lambda_{\mathrm{F}}}{\psi_{0} \sigma^{\prime} \sqrt{2 \pi}} \exp \left(-\frac{\left(\ln \left(\psi_{0}\right)-\mu^{\prime}\right)^{2}}{2 \sigma^{2}}\right) \sum_{l=1}^{\mathrm{L}}\left[\left(1-\mathrm{k}_{\mathrm{f}} \Gamma_{1}^{\delta_{t}} \psi_{0}^{-\delta_{t}} \rho_{\mathrm{F}}\right) \exp \left(-\rho_{\mathrm{F}} \mathrm{k}_{\mathrm{f}} \Gamma_{1}^{\delta_{t}} \psi_{0}^{-\delta_{t}}\right)\right] \mathrm{d} \psi_{0}$

then define $\rho_{\rho_{k+1}} \equiv \rho_{k}+\left.\alpha_{k} \frac{\partial \operatorname{ASE}_{\mathrm{F}}(\rho)}{\partial \rho}\right|_{\rho=\rho_{\mathrm{k}}}$ and $\alpha_{\mathrm{k}+1} \equiv \alpha_{\mathrm{k}}$. Increment the value of $k$ to $k+1$ and iterate Step 2 .

Case II: If $\alpha_{\mathrm{k}} \geq \varepsilon$ and ${ }_{-\mathrm{ASE}_{\mathrm{F}}}\left(\rho_{\mathrm{k}}+\left.\alpha_{\mathrm{k}} \frac{\partial \mathrm{ASE}_{\mathrm{F}}(\rho)}{\partial \rho}\right|_{\rho=\rho_{\mathrm{k}}}\right)>-\operatorname{ASE}_{\mathrm{F}}\left(\rho_{\mathrm{k}}\right)$, then define $\rho_{\mathrm{k}+1} \equiv \rho_{\mathrm{k}}$ and $\alpha_{\mathrm{k}+1} \equiv \frac{\alpha_{\mathrm{k}}}{\mathrm{D}}$. Increment the value of $\mathrm{k}$ to $\mathrm{k}+1$ and iterate step 2 .

If $\alpha_{k}<\varepsilon$, then go to step 3 .

Step 3: Take the value of $\rho_{\mathrm{k}}$ as the optimal solution of Problem (P).

The working principal of the algorithm is as follows. Step 1 is the initialization routine. Step 2 is the main optimization routine. $-\left.\frac{\partial \operatorname{ASE}_{\mathrm{F}}(\rho)}{\partial \rho}\right|_{\rho=\rho_{\mathrm{h}}}$ is the slope of the curve $-\operatorname{ASE}_{\mathrm{F}}(\rho)$ evaluated at $\rho_{\mathrm{k}}$ and $\alpha_{\mathrm{k}}$ relates to the incremental step size between the current evaluation point and the test evaluation point, where the test evaluation point is set at $\rho_{k}+\left.\alpha_{k} \frac{\partial \operatorname{ASE}_{F}(\rho)}{\partial \rho}\right|_{\rho=\rho_{k}}$ and $\alpha_{k}$ is initially set at a large value. If $-\operatorname{ASE}_{\mathrm{F}}\left(\rho_{\mathrm{k}}+\left.\alpha_{\mathrm{k}} \frac{\partial \operatorname{ASE}_{\mathrm{F}}(\rho)}{\partial \rho}\right|_{\rho=\rho_{\mathrm{k}}}\right) \leq-\operatorname{ASE}_{\mathrm{F}}\left(\rho_{\mathrm{k}}\right)$, then the next evaluation point is set at the test evaluation point $\rho_{k+1}=\rho_{k}+\alpha_{k} \frac{\partial \operatorname{ASE}_{\mathrm{F}}(\rho)}{\partial \rho}$ and $\alpha_{k}$ is remained unchanged. It is worth noting that as $-\operatorname{ASE}_{\mathrm{F}}\left(\rho_{\mathrm{k}}+\left.\alpha_{\mathrm{k}} \frac{\partial \operatorname{ASE}_{\mathrm{F}}(\rho)}{\partial \rho}\right|_{\rho=\rho_{\mathrm{k}}}\right) \leq-\operatorname{ASE}_{\mathrm{F}}\left(\rho_{\mathrm{k}}\right)$, the next evaluation point $\rho_{k+1}=\rho_{k}+\left.\alpha_{k} \frac{\partial \operatorname{ASE}_{\mathrm{F}}(\rho)}{\partial \rho}\right|_{\rho=\rho_{k}}$ is moving closer to the optimal solution, because the cost value evaluated at the next evaluation point is smaller than that at the current evaluation point. The incremental step size between two consecutive evaluation points depends on $\alpha_{k}$. As $\alpha_{k}$ is initially set at a large value, the next evaluation point is moving towards the optimal solution significantly. Thereby, the proposed method could significantly improve the convergence rate and reducing the computational costs. It is worth noting that if $-\left.\frac{\partial \operatorname{ASE}_{\mathrm{F}}(\rho)}{\partial \rho}\right|_{\rho=\rho_{\mathrm{k}}}<0 \quad$, then $-\operatorname{ASE}_{\mathrm{F}}\left(\rho_{\mathrm{k}}^{+}\right)<-\operatorname{ASE}_{\mathrm{F}}\left(\rho_{\mathrm{k}}\right) \quad$. If $-\left.\frac{\partial \operatorname{ASE}_{\mathrm{F}}(\rho)}{\partial \rho}\right|_{\rho=\rho_{\mathrm{k}}}>0$, then $-\operatorname{ASE}_{\mathrm{F}}\left(\rho_{\mathrm{k}}^{-}\right)<-\operatorname{ASE}_{\mathrm{F}}\left(\rho_{\mathrm{k}}\right)$. In other words:

$$
-\operatorname{ASE}_{\mathrm{F}}\left(\rho_{\mathrm{k}}+\left.\alpha \frac{\partial \operatorname{ASE}_{\mathrm{F}}(\rho)}{\partial \rho}\right|_{\rho=\rho_{\mathrm{k}}}\right) \leq-\operatorname{ASE}_{\mathrm{F}}\left(\rho_{\mathrm{k}}\right)
$$

if $\quad \alpha \rightarrow 0^{+} \quad$ and $\quad-\left.\frac{\partial \operatorname{ASE}_{\mathrm{F}}(\rho)}{\partial \rho}\right|_{\rho=\rho_{\mathrm{K}}} \neq 0$. However, if $-\operatorname{ASE}_{\mathrm{F}}\left(\rho_{\mathrm{k}}+\left.\alpha_{\mathrm{k}} \frac{\partial \operatorname{ASE}_{\mathrm{F}}(\rho)}{\partial \rho}\right|_{\rho=\rho_{\mathrm{k}}}\right)>-\operatorname{ASE}_{\mathrm{F}}\left(\rho_{\mathrm{k}}\right)$, then it implies that the test evaluation point has been moved beyond the optimal solution. Hence, the next evaluation point is set at the current evaluation point, that is $\rho_{\mathrm{k}+1}=\rho_{\mathrm{k}}$, but $\alpha_{\mathrm{k}}$ is reduced according to $\alpha_{k+1}=\frac{\alpha_{k}}{D}$, in which $D>1$.

By iterating the above procedures, the optimal solution can be reached efficiently. Finally, if $\alpha_{k}$ is reduced to a very small value such that $\alpha_{k}<\varepsilon$, then the algorithm terminates and we can assume that the optimal solution is reached.

In order not to terminate the algorithm when the convergence rate of the algorithm is slow and to have a high accuracy of the solution, $\varepsilon$ should be chosen at a small value and $\mathrm{D}$ should be chosen as a positive number larger than but close to 1 . We choose, $\varepsilon=10^{-6}$ and $\mathrm{D}=$ 1.0001 which are good enough for most applications. 
The initial search point of the optimization algorithm $\rho_{1}$ is set at the midpoint of the range of $\rho_{\mathrm{F}}$, that is $\rho_{1}=0.5$. As $\alpha_{1}$ should be set to a large value initially, we set it as 0.1 , which is good enough for most applications.

\section{RESULTS}

For a fair comparison of our proposed method with the exhaustive search method (with 100 searching points) (Chandrasekhar and Andrews, 2009), the same set of parameter values used in (Chandrasekhar and Andrews, 2009), are employed in our simulations. That is, $\mathrm{L}=8, \quad \delta_{\mathrm{f}}=0.5714, \Gamma_{l}=\left(2^{l}-1\right) \times 10^{0.3}$ for $1=1,2, \ldots, 8$, $\mu=0 \mathrm{~dB}, \sigma^{2}=4 \mathrm{~dB}, \mu_{\mathrm{i}}=0 \mathrm{~dB} \forall_{\mathrm{i}} \in \Phi, \sigma_{\mathrm{i}}^{2}=12 \mathrm{~dB} \forall_{\mathrm{i}} \in \Phi, \mathrm{r}_{\mathrm{F}}=40$ and $r_{M}=288$ for $N_{f}=10,50,100$. Based on these sets of parameters, we ran the proposed adaptive gradient vector step size algorithm and obtain optimal values of $\rho_{\mathrm{F}}$ as shown in Fig. 1, i.e., $\rho_{\mathrm{F}}=1$ for $\mathrm{N}_{\mathrm{f}}=10, \rho_{\mathrm{F}}=0.55$ for $\mathrm{N}_{\mathrm{f}}=50$ and $\rho_{\mathrm{F}}=0.3$ for $\mathrm{N}_{\mathrm{f}}=100$, which are the same as those obtained by exhaustive searches in (Chandrasekhar and Andrews, 2009). It is worth noting that there is only one single stationary point in the curves shown in the figure. This is because of the nature of the probability density functions. However, in terms of the computational cost, it takes only 4.5687 $\mathrm{sec}$ for running our proposed algorithm for these three cases, while it takes $7.8321 \mathrm{~min}$ for running the exhaustive search method (Chandrasekhar and Andrews, 2009) under the same computer setup in a computer with the AMD Athlon(tm) II X4 630 Processor 2.8GHz, 6.00GB RAM and 64-bit operating system (windows 7 Home Premium). Hence, it can be concluded that our proposed method is more efficient than the exhaustive search method.

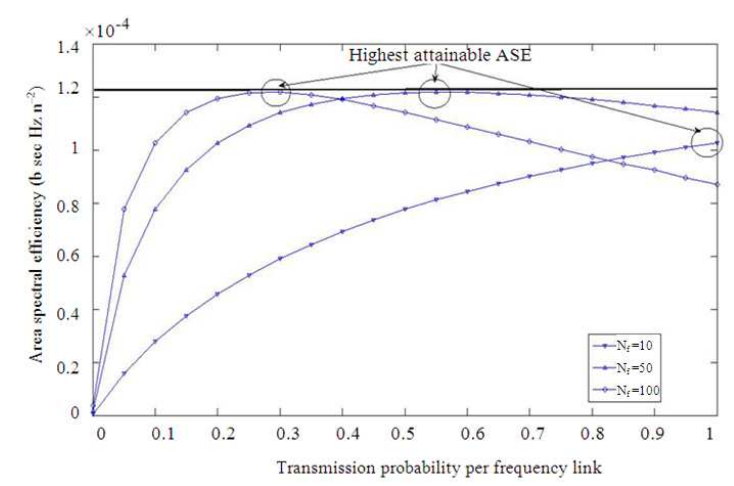

Fig. 1: The relationship between the ASE of femtocells and the fraction of radio resources per transmission interval

\section{DISCUSSION}

Figure 1 shows the different strategies that can be adopted in order to maximize the ASE. For a small number of femtocells in the network $\left(\mathrm{N}_{\mathrm{f}}=10\right)$, it is best for each femtocell to access the entire allocated spectrum $\left(\rho_{\mathrm{F}}=1\right)$. On the other hand, the higher the number of femtocells in the network $\left(\mathrm{N}_{\mathrm{f}}=100\right)$, the lower the percentage of accessible allocated spectrum for each femtocell $\left(\rho_{\mathrm{F}}=0.3\right)$ is required in order to maximize the ASE.

\section{CONCLUSION}

In this study, an adaptive gradient vector step size approach is proposed for finding the optimal solution of the decentralized resource allocation scheme for the OFDMA downlink of a femto network. The ASE is maximized subject to a constraint on the radio resource per transmission interval accessible by each femtocell. Based on the numerical computer simulation results, it can be concluded that the computational costs of our proposed method are much lower than that of the existing exhaustive search methods.

\section{ACKNOWLEDGEMENT}

Study was supported by the UK EPSRC grant CASE/CNA/07/106.

\section{REFERENCES}

Andrews, J.G., 2008. Fundamentals of WiMAX: Understanding Broadband Wireless Networking. 1st Edn., Pearson Education India, India, ISBN: 10: 8131726355 , pp: 496.

Assaad, M. and A. Mourad, 2008. New frequency-time scheduling algorithms for 3GPP/LTE-like OFDMA air interface in the downlink. Proceedings of the IEEE Vehicular Technology Conference, May 11-14, IEEE Xplore Press, Singapore, pp: 1964-1969. DOI: 10.1109/VETECS.2008.444

Chandrasekhar, V., J. Andrews and A. Gatherer, 2008. Femtocell networks: A survey. IEEE Commun. Mag., 46: 59-67. DOI: 10.1109/MCOM.2008.4623708

Chandrasekhar, V. and J. Andrews, 2009. Spectrum allocation in tiered cellular networks. IEEE Trans. Commun., 57: 3059-3068. DOI: 10.1109/TCOMM.2009.10.080529

Chandrasekhar, V., M. Kountouris and J.G. Andrews, 2009. Coverage in multi-antenna two-tier networks. IEEE Trans. Wireless Commun., 8: 5314-5327. DOI: 10.1109/TWC.2009.090241 
Am. J. Engg. \& Applied Sci., 4 (4): 491-496, 2011

Elayoubi, S.E., O.B. Haddada and B. Fouresti, 2008. Performance evaluation of frequency planning schemes in OFDMA-based networks. IEEE Trans. Wireless Commun., 7: 1623-1633. DOI: 10.1109/TWC.2008.060458

Lopez-Perez, D., G.D.L. Roche, A. Valcarce, A. Juttner and J. Zhang, 2008. Interference avoidance and dynamic frequency planning for WiMAX femtocells networks. Proceedings of the 11th IEEE Singapore International Conference on Communication Systems, Nov. 19-21, IEEE Xplore Press, Guangzhou, pp: 1579-1584. DOI: 10.1109/ICCS.2008.4737449

Necker, M.C., 2007. Local interference coordination in cellular OFDMA networks. Proceedings of the IEEE 66th Vehicular Technology Conference, Sept. 30-Oct. 3, IEEE Xplore Prees, Baltimore, MD., $\quad$ pp: 1741-1746. DOI: 10.1109/VETECF.2007.368

Simonsson, A., 2007. Frequency reuse and intercell interference co-ordination in E-UTRA. Proceedings of the IEEE 65th Vehicular Technology Conference, Apr. 22-25, IEEE Xplore Press, Dublin, pp: 3091-3095. DOI: 10.1109/VETECS.2007.633
Stolyar, A.L. and H. Viswanathan, 2008. Selforganizing dynamic fractional frequency reuse in OFDMA systems. Proceedings of the IEEE 27th Conference on Computer Communications, Apr. 13-18, IEEE Xplore Press, Phoenix, AZ, pp: 691699. DOI: 10.1109/INFOCOM.2008.119

Turkmani, A.M.D., 1992. Probability of error for Mbranch macroscopic selection diversity. IEE Proc. Commun. Speech Vision, 139: 71-78.

Weber, S., J.G. Andrews and N. Jindal, 2007. The effect of fading, channel inversion and threshold scheduling on ad hoc networks. IEEE Trans. Inform. Theory, 53: 4127-4149. DOI: 10.1109/TIT.2007.907482

Yeh, S.P., S. Talwar, S.C. Lee and H. Kim, 2008. WiMAX femtocells: A perspective on network architecture, capacity and coverage. IEEE Commun. Mag., 46: 58-65. DOI: 10.1109/MCOM.2008.4644120 